Int. J. Dev. Biol. 48: 889-902 (2004)

doi: $10.1387 / \mathrm{ijdb} .041880 \mathrm{jk}$

\title{
Development of lens sutures
}

\author{
JER R. KUSZAK*,1, REBECCA K. ZOLTOSKI ${ }^{2}$ and CLIFFORD E. TIEDEMANN ${ }^{3}$ \\ ${ }^{1}$ Departments of Ophthalmology and Pathology, Rush University Medical Center, ${ }^{2}$ Department of Basic and Health Sciences, Illinois College \\ of Optometry, Chicago, and ${ }^{3}$ Department of Anthropology, University of Illinois at Chicago, Chicago, IL USA
}

\begin{abstract}
Cylindrical map projections (CMPs) have been used for centuries as an effective means of plotting the features of a 3D spheroidal surfaces (e.g. the earth) on a 2D rectangular map. We have used CMPs to plot primate fiber cell organization from selected growth shells as a function of growth, development and aging. Lens structural parameters and features were derived from slitlamp, light and transmission and scanning electron micrographs. This information was then used to create CMPs of lenses that were then correlated with azimuthal map projections (AMPs; projections that are radially symmetric around a central point [the poles] ) to reveal different suture patterns during distinct time periods. In this manner, both lens fiber and suture branch locations are defined by degrees of longitude and latitude. CMPs and AMPs confirm that throughout defined periods of development, growth and ageing, increasingly complex suture patterns are formed by the precise ordering of straight and opposite end curvature fibers. However, the manner in which additional suture branches are formed anteriorly and posteriorly is not identical. Anteriorly, new branches are added between extant branches. Posteriorly, pairs of new branches are formed that progressively overlay extant branches. The advantage of using CMPs is that the shape and organization of every fiber in a growth shell can be observed in a single image. Thus, the use of CMPs to plot primate fiber cell organization has revealed more complex aspects of fiber formation that may explain, at least in part, changes in lens optical quality as a function of age and pathology. In addition, more accurate measurements of fiber length will be possible by incorporating the latitudinal and longitudinal locations of fibers.
\end{abstract}

KEY WORDS: crystalline lens, cylindrical map projection, azimuthal map projection, suture development, fiber cell organization, $3 D C A D$

\section{Introduction}

The crystalline lens of the eye is a principal component in the process of dynamic focusing or accommodation. To perform its role in accommodation, the lens must be transparent and also have the capacity to rapidly alter its shape as it transitions between focusing on near and distant objects. Underlying lens function is lens structure.

Gross (light and scanning confocal microscopy) and ultrastructural (scanning, transmission and freeze-etch electron microscopy) analysis of all vertebrate lenses reveals that lenses are composed of exceedingly long fiber-like cells that are of uniform cross-sectional shape (hexagonal) and size (Cohen, 1965; Smelser, 1965; Kuszak and Rae, 1982; Rae et al., 1982; Rae et al., 1983; Kuszak et al., 1985; Kuszak et al., 1996; Al-Ghoul and Costello, 1997; Bassnett and Winzenburger, 2003). These microscopic techniques also show that in general as these fibers are formed throughout life, they are overlain, in register, as age-related concentric growth shells. Thus, it has been proposed that the highly ordered arrangement of lens fibers contributes to lens transparency by transforming the individual fibers into a series of coaxial refractive surfaces (Trokel, 1962). As a result, large particle scatter is minimized as light is transmitted through the membranes of hundreds of thousands of fibers. Small particle scatter is minimized as light is transmitted through an age-related gradient of refractive index established and maintained by the cytoplasmic crystalline proteins of fibers.

However, while the structure of all vertebrate lenses is similar, it is not identical. Different sutures, the end to end arrangement of

Abbreviations used in this paper: AMP, azimuthal map projection; CAD, computer assisted drawing; CMP, cylindrical map projection.

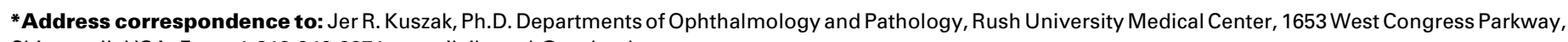
Chicago, IL USA. Fax: +1-312-942-2371. e-mail: jkuszak@rush.edu

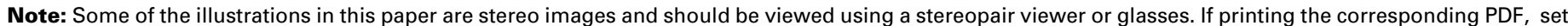
your printer to print at $100 \%$ rather than to fit page, as distorsions in the proportions of the stereopairs can occur otherwise. 

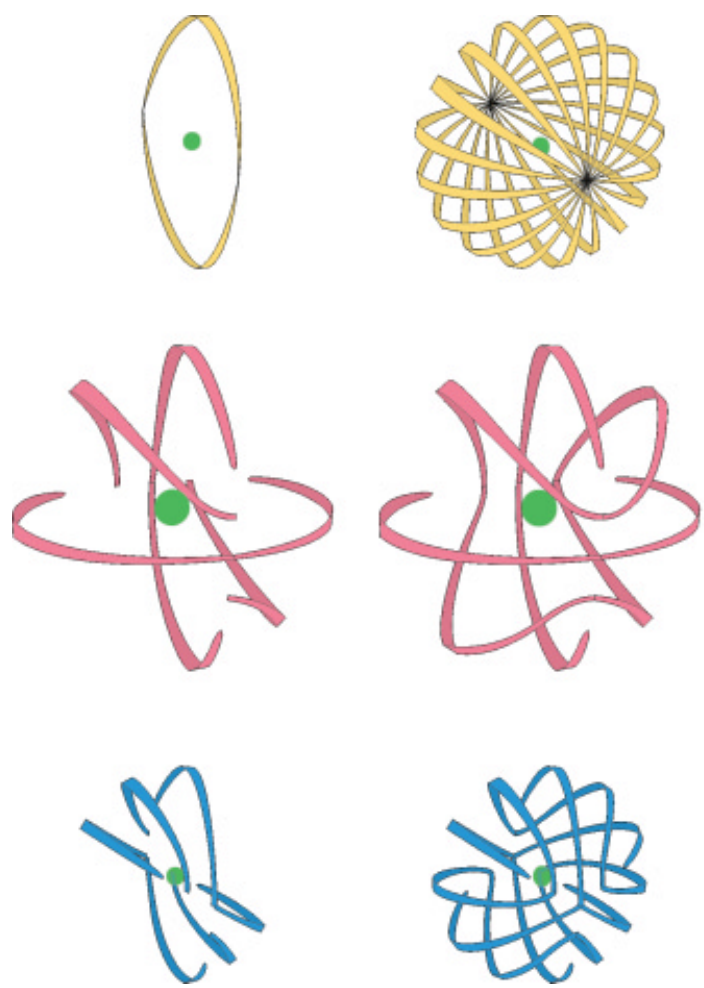
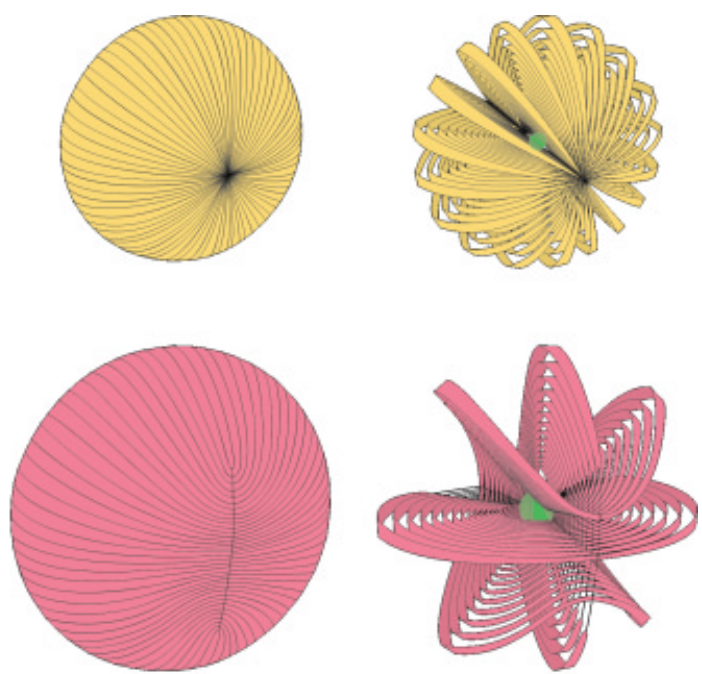

Fig. 1. Scale (5x) 3D CADs showing fiber shape and arrangement in avian and reptilian (top row), rabbit and amphibian (second row) and rodent, feline, canine, porcine, ovine, and bovine (bottom row) lenses, (first column, top to bottom). While avian and reptilian lenses have meridian-like fibers, all other lenses have straight and curved fibers. (second and third columns, top to bottom) As a result avian and reptilian fibers, positioned on opposite sides of the equator, are arranged end to end in growth shells to form anterior and posterior "umbilical" sutures. In all other lenses, curved fibers, organized as enantiomeric groups that bracket straight fibers, are arranged end to end in growth shells to form "line" and " $Y$ " suture branches. (fourth column, top to bottom) While there is no intra-shell variation in fiber shape in avian and reptilian lenses, there is an intra-shell variation in fiber shape in all other lenses. However, there is no inter-shell variation in fiber shape in umbilical, line, or Y suture lenses. Consequently, in these lenses intershell fiber length varies as a simple radial function.

non-uniform fiber ends within and between growth shells, define four distinct types of lenses. It is important to recognize this fact because each type of suture exerts a different quantifiable negative influence on lens optical quality (Kuszak et al., 1991; Sivak et al., 1994; Kuszak etal., 1994; Priolo etal., 1999). In addition, abnormal development of sutures has been shown to be associated with specific types of cataract (Al-Ghoul etal., 1999; Kuszak etal., 1999; Kelz et al., 2000). Finally, while some ocular surgeries (trabeculectomy and vitrectomy) result in post-surgical alterations in lens sutures that precede cataract formation, these detrimental sutural changes may be preventable (Kuszak et al., 2000; Kuszak et al., 2002).

Therefore, while other contributors to this edition will review important aspects of development and differentiation as they apply to all vertebrate lenses, this review will describe the culmination of these processes, specifically in regards to the development of sutures during gestation, infancy, adolescence through adulthood and adulthood through middle age in humans.

\section{Lens developmental anatomy}

\section{Primary fibers}

The basic structure of all vertebrate lenses is established early in development (O'Rahilly and Meyer, 1959; Rafferty and Esson,
1974; Kuszak et al., 1980; Kuszak and Rae, 1982; Kuszak et al., 1985; Rafferty, 1985; Kuszak et al., 1996; Kuszak and Costello, 2002). Lens formation begins as surface ectodermal cells, immediately overlying the developing optic vesicle, are induced to thicken and form the lens placode. As the optic vesicle folds inward to form the optic cup, the lens placode invaginates toward the developing optic cup until it eventually pinches off as an inverted (inside-out) lens vesicle. Lens development proceeds as cells of the lens vesicle approximating its retinal half are induced to terminally differentiate. The most apparent structural consequence of lens terminal differentiation is that the originally cuboidal lens vesicle cells, elongate into long fiber-like cells, or fibers. This initial, or "primary fiber" formation, proceeds until the lumen of the lens vesicle is nearly obliterated.

\section{Secondary Fibers}

The cells of the lens vesicle that were not induced to form primary fibers remain as a monolayer, the lens epithelium, that covers the anterior surface of the primary fiber mass. From this point on further lens development and growth occurs throughout life, in a manner similar to other stratified epithelia (Harding et al., 1971). The lens epithelium constitutes the basal layer. However, whereas typically stratified epithelia have their progenitor cells distributed throughout the basal layer, the lens is unique in that its 

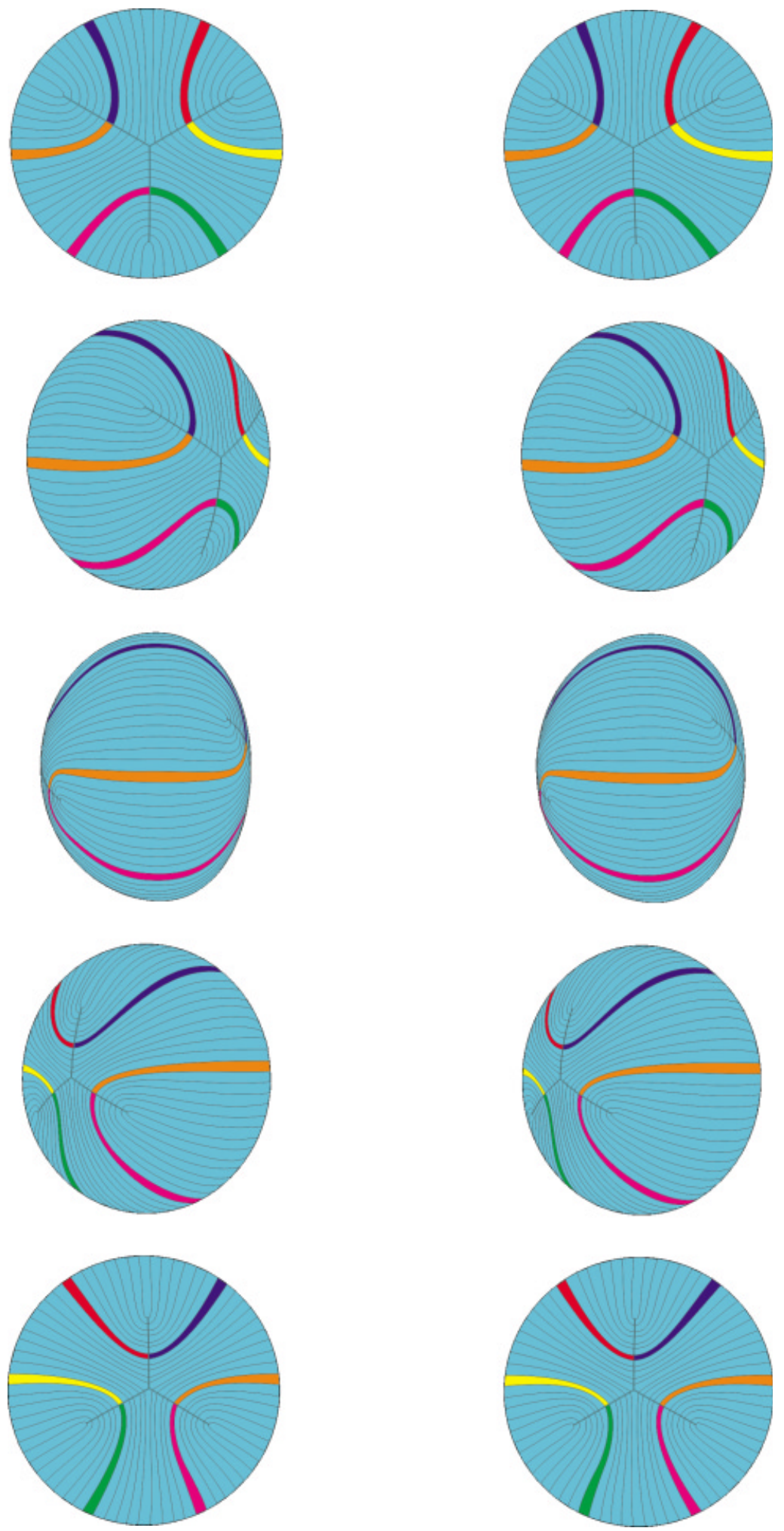

progenitor cells are sequestered as a distinct sub-population within the lens epithelium known as the germinative zone (GZ). GZ cells comprise a narrow, peripheral, latitudinal band of the lens epithelium located just above the equator. These cells undergo mitotic division and selected daughter cells are induced to terminally differentiate and form "secondary fibers".

As with primary fiber formation, the most apparent structural consequence of secondary fiber formation is the transformation of a cuboidal cell into a long fiber. However, while forming primary fibers are fixed in position as they elongate essentially uni-directionally, secondary fiber formation requires the forming fibers to rotate about their polar axis while simultaneously migrating posteriorly and elongating bidirectionally. Fiber rotation is complete when the long axis of a forming fiber is aligned parallel to the antero-posterior axis of the lens. Fiber migration is complete when the center of a forming fiber reaches the mid-point between the poles which by virtue of the fact that all vertebrate lenses are asymmetric, oblate spheroids, is posterior to the equator. As secondary fibers elongate, their anterior ends are insinuated between the lens epithelium and the primary fiber mass while at the same time their posterior ends are insinuated between the primary fiber mass and the posterior lens capsule. Secondary fiber elongation is complete when the anterior and posterior ends of newly formed secondary fibers break contact with respectively, the apical surfaces of epithelial cells anteriorly, and the capsule posteriorly. At that point, the anterior ends of defined pairs of fibers abut and overlap with one another, as do their posterior ends, to form "sutures" thereby completing a growth shell.

\section{Lens sutural anatomy}

Avian and reptilian fibers are meridians, crescent cells with their entire length lying in a plane that passes through the antero-posterior axis defined by their equatorial position (Fig. 1, first row) (Duke-Elder, 1970; Kuszak et al., 1980; Willekens and Vrensen, 1985; Bassnett and Winzenburger, 2003). As fiber development and growth occurs circumferentially around the equator, fibers from opposite sides of avian and reptilian lenses become arranged end to end as pairs forming ellipses around the embryonic nucleus. By extrapolation, the growth shells of these lenses consist of the accumulation of paired fibers about the antero-posterior axis. The end to end arrangement of fibers within and between growth shells anteriorly and posteriorly are referred to respectively as the anterior and posterior "umbilical" sutures.

In all other lenses, fibers are partial or incomplete meridians. That is to say upon completion of elongation, the vast majority of fibers do not have ends that extend to

Fig. 2. A series of scale (5x) 3D CAD stereopairs showing the formation of anterior $\mathbf{Y}$ and offset posterior $\mathbf{Y}$ sutures. (First row) In this view of the anterior surface, a number of enantiomeric curved fibers have been highlighted in red, yellow, green, magenta, orange, and purple. The anterior ends of these fibers are paired (red and yellow, green and magenta, and orange and purple) to form parts of anterior suture branches. By following these fibers along their length (second through fifth rows, at respectively 45, 90, 135, and $180^{\circ}$ to the anterior polar axis), it can be seen that as a result of opposite end curvature, the posterior ends of these fibers are paired with different fibers (purple and red, yellow and green, and magenta and orange) to form offset posterior suture branches. 

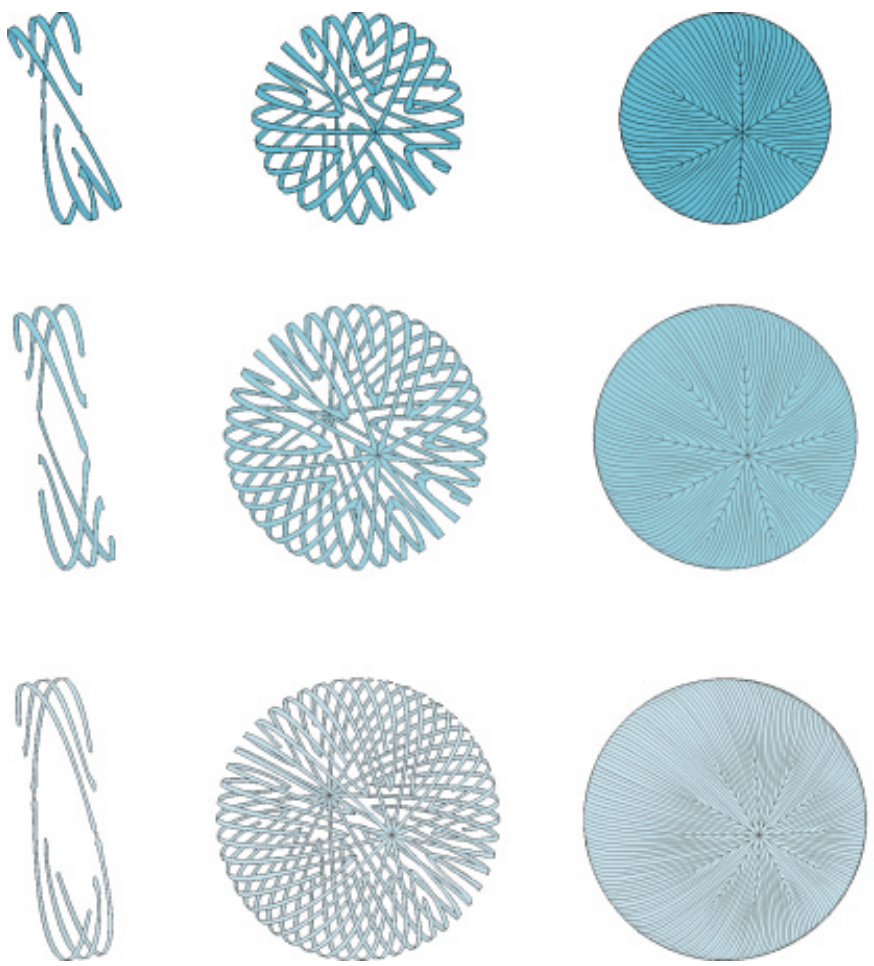

Fig. 3. Scale (4x) 3D CADs showing fiber shape and arrangement in infantile (top row), adolescent (second row) and adult (bottom row) human lenses, (first column, top to bottom). Human lenses are also composed of straight and curved fibers. (Second and third columns, top to bottom) With each successive stage of development and growth, there is an increased number of straight fibers. As a result, curved fibers, with progressively less end curvature, and organized as more numerous enantiomeric groups that bracket straight fibers, are arranged end to end in growth shells to form "simple star", "star" and "complex star" sutures. (Fourth column, top to bottom) The range of intra-shell variation in fiber shape increases with each successive stage of development and growth. In addition, the inter-shell variation in fiber length is not a simple radial function.

the poles (Fig. 1, second - third rows). In addition, most fibers only have their middle portion, or mid-segment, that lies in a plane that passes through the antero-posterior axis of a lens defined by their equatorial position. The end portions, or anterior and posterior end segments of most fibers, generally curve to a varying extent, in opposite directions away from the polar axis. Thus, while all fibers exhibit crescent curvature, most fibers are also S-shaped along their length. It is this latter curvature that is referred to when describing a fiber as a "curved fiber".

However, a small number of fibers do have one of their ends that extends to a lens pole. The entire length of these fibers does lie in a plane that passes through the antero-posterior axis of a lens defined by their equatorial position. Although these fibers exhibit crescent curvature along their length, it is their lack of end curvature that is referred to when describing these fibers as "straight fibers".

Therefore, in most vertebrate lenses, fibers positioned on opposite sides of the equator "do not" become arranged end to end as pairs forming ellipses around the embryonic nucleus to make sutures that complete a growth shell. Instead which fibers do become arranged end to end as pairs to form sutures is directly related to the equatorial position straight fibers. In
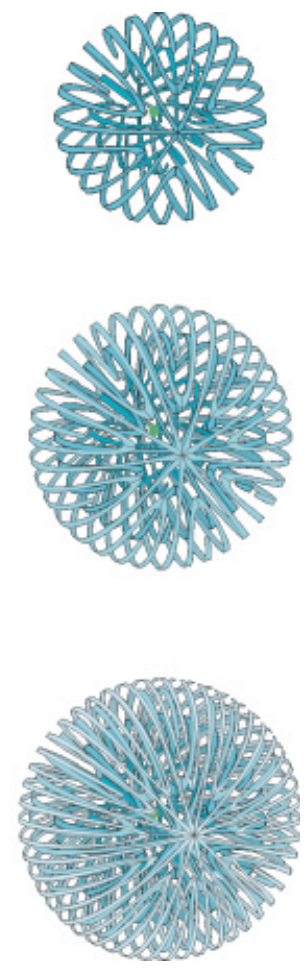

addition, each curved fiber is paired with two other fibers (Fig. 2). The anterior end of a curved fiber becomes arranged end-to-end with the anterior end of its defined paired fiber to form part of an anterior suture branch, within the anterior half of a growth shell. The posterior ends of these paired fibers become arranged end-to-end with the posterior ends of two other defined paired fibers to form parts of two offset posterior suture branches within the posterior half of the same growth shell. In this manner, line and $Y$ suture patterns are formed that characterize respectively, amphibian and rabbit, and rodent, canine, feline, porcine, ovine and bovine lenses.

\section{D CAD and cylindrical map analysis of human lens suture anatomy}

As shown in Figs. 1 and 2, lens sutures are three-dimensional (3D) entities formed within and between growth shells. Furthermore, since the posterior curvature of lenses is greater than the anterior curvature, the three-dimensionality of anterior and posterior sutures are unequal. Thus, the commonly used methodologies of thick and thinsectioning of embedded tissue for light and transmission electron microscopy analysis, are not very effective for examination of lens suture anatomy because by definition these are planar techniques. Frontal sections (perpendicular to the polar axis) through lenses fail to include either complete anterior or posterior suture patterns in any lens. Similarly, while sagittal and para-sagittal sections (parallel to the polar axis) through lenses in theory could include an entire suture branch, at least from a small lens (mouse), the precise orientation of an embedded lens necessary to produce such a microtome section is at best very difficult. And, as will be demonstrated later, such sections would also be ineffective in revealing the progressively more complex suture patterns formed in primate lenses throughout life.

It is more useful to employ morphological techniques that either preserve, or adequately represent, lens suture anatomy in the third dimension as they exist in situ. Analysis of lenses dissected at age-defined depths by scanning electron microscopy correlated with 3D computer assisted drawing (CAD) reconstructions, are effective means for examination of lens suture anatomy. In a scanning electron microscope a lens can be mounted on either of its poles so that the entire opposite surface, with a complete and intact suture pattern, is exposed for examination. Then by tilting a lens in appropriate \pm amounts, stereomicrographs can be produced that accurately represent 
the three dimensionality of a suture pattern. From such stereomicrographs complete growth shells with both anterior and posterior suture patterns exposed can be reproduced in scale 3D CAD reconstructions (Figs. 1-3).

\section{Cylindrical map projections of suture development dur- ing gestation, growth and aging}

As demonstrated in Fig. 1, the following general principles apply to umbilical, line and $Y$ lens sutures. Each successive growth shell contains the same number of straight fibers in identical equatorial locations. Each successive growth shell contains an equal number of curved fiber groups in identical equatorial locations. Each successive growth shell contains an equal number of suture branches in identical equatorial locations. Thus in most lenses, the essential suture parameters are represented in any one growth shell. By comparison, the essential suture parameters of primate lenses are not represented in any one growth shell, because these lenses form progressively more complex iterations of sutures during defined periods of development, growth, and aging (Fig. 3). Therefore, to facilitate an understanding of primate lens suture development during gestation, growth, and aging, we employ, cylindrical map projections.

Cylindrical map projections (CMPs) are a method of represent- ing a curved 3D surface, like the earth, onto a 2D map. Geradus Mercator devised the first CMPs of the earth in 1569, for use in navigation. Described simplistically, a cylinder is wrapped around the circumference of a spheroid (e.g. the earth or a lens) and the details of the surface are then projected onto the interior of the cylinder. The cylinder is then unwrapped to produce a rectangular map that permits direct, simultaneous observation of points from opposite sides of a globe. Since the lens, like the earth, is an asymmetrical oblate spheroid, it lends itself to cylindrical map analysis (Fig. 4). Consider the following; the earth consists of unequal northern and southern hemispheres. The hemispheres span the distance from $0^{\circ}$ latitude at the equator to, respectively, $90^{\circ}$ and $-90^{\circ}$ latitude at the north and south poles. Lenses feature unequal anterior and posterior surfaces that span the distance from their equators to their anterior and posterior poles. Distances and position around the earth's equator are defined by degrees of longitude beginning at the prime meridian ( $0^{\circ}$ longitude) and extending between $+180^{\circ}$ (east) and $-180^{\circ}$ (west). Of course lenses do not feature a prime meridian; therefore, by convention we describe the meridian-like fibers positioned around a lens equator beginning at the most superior point of a lens in the anatomical position, and extending $360^{\circ}$ around the equatorial circumference. In this manner, in an OD lens 0 to 90,90 to 180 , 180 to 270 , and 270 to $360^{\circ}$ longitude define respectively the
Fig. 4. A comparison of the basic parameters of cylindrical map projections of the earth and crystalline lenses. (Upper) The northern and southern hemispheres of the earth and the anterior and posterior surfaces of lenses are mapped from $0^{\circ}$ latitude at the equator to \pm 90 latitudinal degrees at the poles. The equatorial circumference of the earth is mapped from the prime meridian, $0^{\circ}$ longitude, to $180^{\circ}$ east longitude and $-180^{\circ}$ west longitude. (Lower) By convention we mapped the equatorial circumference of an $O D$ lens from its most superior aspect in the anatomical position, $0^{\circ}$ longitude, to $90,180,270$ and $360^{\circ}$ longitude spanning respectively, the supero-nasal, infero-nasal, inferotemporal, and supero-temporal quadrants of the lens. The mirror image OS lens spans the same quadrants in reverse order. The width and height of the map is determined by the size of the lens. The position of the lines of latitude are based on established mathematical equations that measure the distance between two points on a curved surface. Because lenses are asymmetrical oblate spheroids, several features are immediately apparent. First, the area of the anterior surface (top portion of the lower map) of the lens is less than that of the posterior surface (bottom portion of the lower map). Additionally, the distance from the 0 to $30^{\circ}$ latitudinal line is less than that from the 30 to $60^{\circ}$, which is less than the distance between the 60 and $90^{\circ}$ latitudinal lines. This leads to a visual distortion of the fibers at the poles, as they widen to form the sutures (shown in black). Lastly, the lines of latitude are positioned differently on the respective surfaces. This and subsequent cylindrical map projections of lenses have been simplified by removing every other group of fibers, as well as color coding the radial location of fibers (embryonic to adult dark to light blue) and suture branches (fetal to adult yellow to orange [anterior] and light to dark red [posterior]). Because the cylindrical map projections unwraps the suture patterns, each map is supplemented by a simple polar projection showing the anterior and offset posterior sutures.

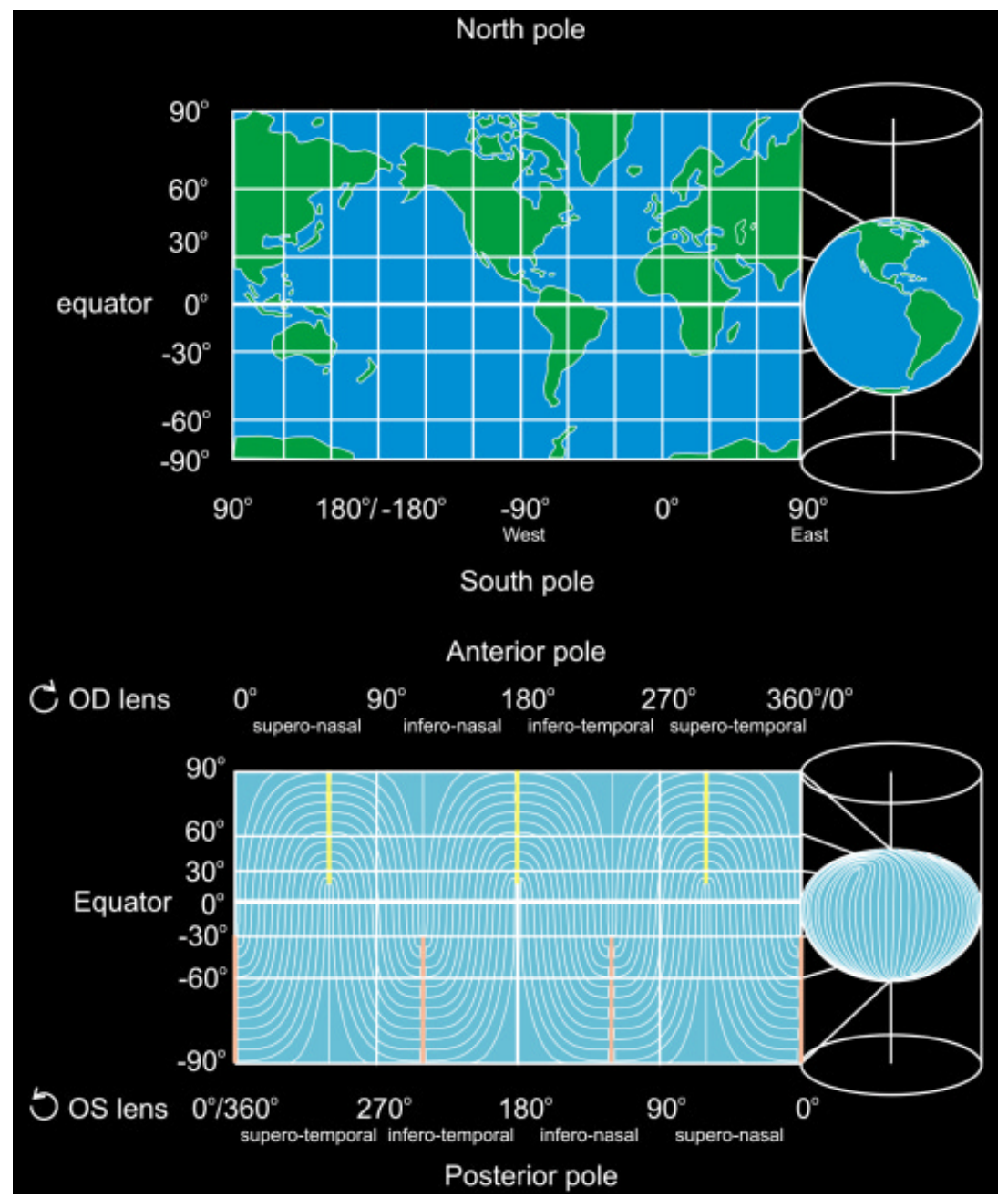




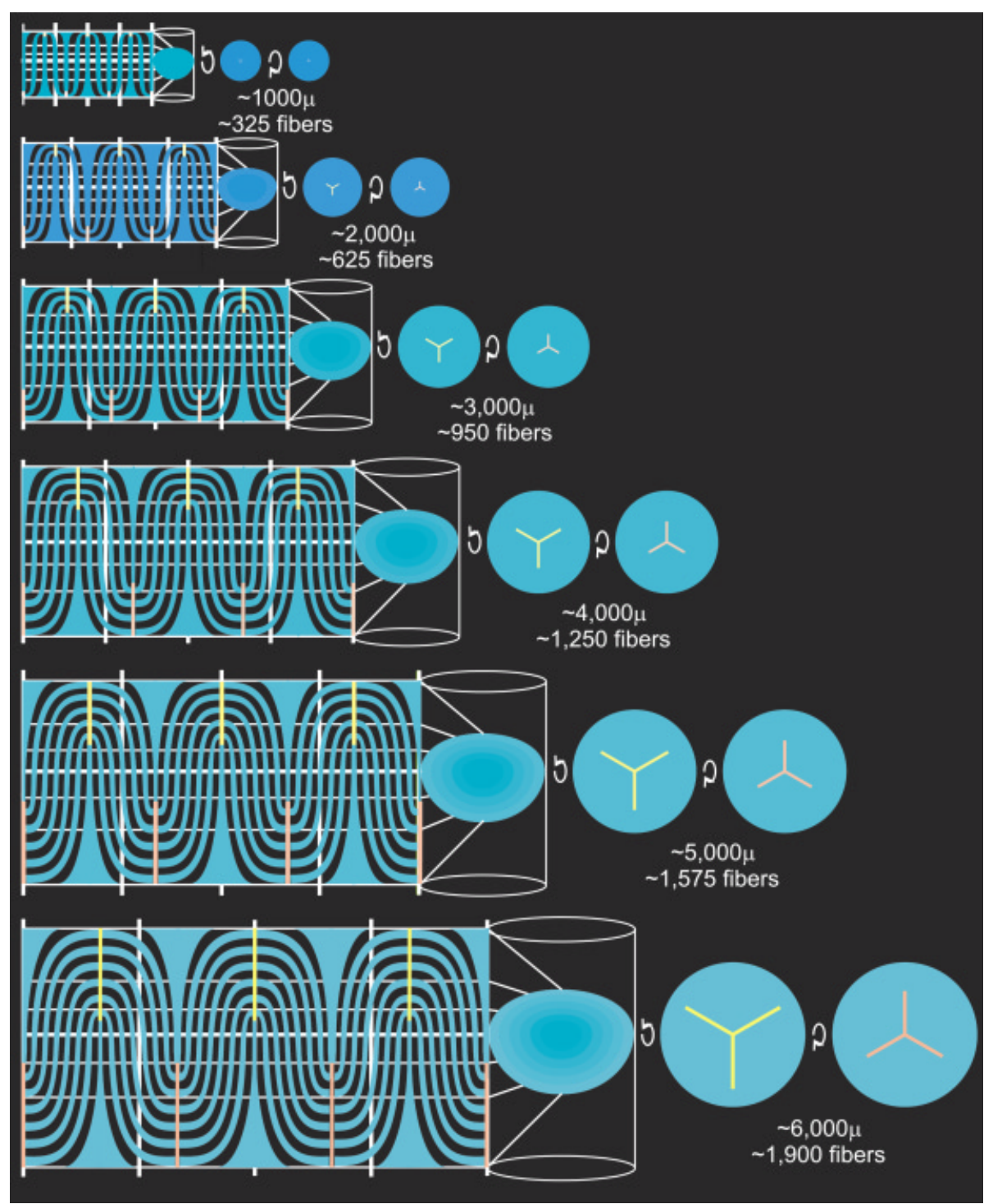

Fig. 5. A series of cylindrical map and polar projections demonstrating the formation of $\mathbf{Y}$ sutures throughout gestation. The straight fibers define the origin and termination of primary suture branches. The end to end arrangement of enantiomeric curved fiber groups form the anterior and posterior sutures.

supero-nasal, infero-nasal, infero-temporal, and supero-temporal quadrants as viewed along the polar axis from the anterior surface. The OS lens is the enantiomer of the OD lens. A representative CMP of the outermost mature growth shell (located $\sim 3,000 \mu \mathrm{m}$ from the lens center in the equatorial plane) from a primate lens at birth is shown in Fig. 4, bottom. In this and each subsequent map, each fiber shape represents a group of $\sim 20$ fibers (estimates of the \# of fibers/growth shell are calculated as follows: circumference $[C]=\pi$ growth shell diameter $[D] /$ avg.fiber width). Note that the organization of each fiber and suture branch, including variable length and curvature is quantifiably revealed in a single CMP. To further facilitate an appreciation for fiber organization, every other group of fibers has been eliminated. In addition, both fibers and sutures are color coded in successive CMPs (Figs. 5 - 8) to help distinguish the differences in fiber length and curvature in concentric growth that result in more complex sutures being formed throughout life. Subtle but perceptible hues of dark to light blue are used to distinguish fiber age (older to younger). Similarly, subtle but perceptible hues of respectively yellow to orange and light to dark red are used to distinguish anterior and posterior suture branches (older to younger).

However, a major disadvantage of using CMPs for sutural analysis is that by definition they unravel the characteristic $Y$ and star shapes of suture patterns. Therefore, each CMP is supplemented by modified polar stereographic maps showing the relationship between anterior and posterior suture patterns in age-defined growth shells. Finally further modified polar stereographic maps of suture patterns between growth shells will be used to demonstrate the formation of discontinuous suture planes throughout life (Figs. 9 and 10).

Y Suture Formation During Gestation: Primary fibers are neither uniform in shape nor size (Rae et al., 1982; Kuszak et al., 1985; Kuszak et al., 1989; Al-Ghoul and Costello, 1997; Bassnett and Winzenburger, 2003). As such, the primary fiber cell mass, or embryonic lens nucleus, does not consist of growth shells overlain in register to form ordered radial cell columns. The initial secondary fibers are similarly non-uniform in shape and size and also lack an ordered arrangement. Only as lens development proceeds are the additional secondary fibers formed progressively more uniform in shape. The establishment of growth shells comprised of uniform fibers overlain in register as radial cell columns occurs within 250 - 750 $\mu \mathrm{M}$ of the equatorial center of the lens. Coincidentally, this marks the beginning of suture formation.

The formation of $Y$ sutures throughout human development is shown in Fig. 5 . The growth shell located approximately $500 \mu \mathrm{M}$ from the equatorial center of a human lens consists of $\sim 325$ fibers. Six of these fibers are straight fibers. The three straight fibers positioned at 60,180 and $300^{\circ}$ of longitude, have a posterior end that extends to the posterior pole, but an anterior end that does not extend to the anterior pole. By convention these fibers are referred to as posterior straight fibers (PSFs). The other three straight fibers, positioned at 0,120 and $240^{\circ}$ of longitude, have an anterior end that extends to the anterior pole, but a posterior end that does not extend to the posterior pole. By convention these fibers are referred to as anterior straight fibers (ASFs). Thus, as fiber differentiation produced this fetal growth shell, short, longitudinal arc length gaps were created between the anterior ends of PSFs and the anterior pole, and between the posterior ends of ASFs and the posterior pole. The remaining $300+$ curved fibers of this growth shell are in six equal groups bracketing straight fibers. As these 
fiber elongated, their ends curved in opposite directions away from the polar axis until they filled the longitudinal arc length gaps between the poles and the ends of straight fibers. The amount of end curvature/individual fiber varies relative to its position to straight fibers (end curvature of fibers located near straight fibers $>$ end curvature of fibers located a distance from straight fibers). The precise end-to-end arrangement of the curved fibers constitutes the original $Y$ suture. The anterior ends of curved fibers that bracket PSFs abut and overlap to form three anterior suture branches. Simultaneously, the posterior ends of the same curved fibers that bracket ASFs, as a consequence of opposite end curvature, abut and overlap to form three, offset posterior suture branches.

As additional fibers are added throughout gestation ( 1,900 fibers in the outermost mature fetal growth shell at birth), each successive growth shell still contains three PSFs and three ASFs positioned at respectively, 60,180 and 300 , and 0,120 and $240^{\circ}$ longitude. Therefore, the length of suture branches increases as radially longer, but identically shaped fibers are overlain in register onto successive growth shells.

Thus, as regards $Y$ suture formation during gestation, the following fiber differentiation parameters normally apply:

The anterior ends of PSFs define the proximal ends, or origins of anterior suture branches.

The posterior ends of PSFs define the distal ends, or terminations of posterior suture branches.

The posterior ends of ASFs define the proximal ends, or origins of posterior suture branches.

The anterior ends of ASFs define the distal ends, or terminations of anterior suture branches.

There is an intra-shell variation in curved fiber shape that is directly related to the closest straight fiber.

There is no inter-shell variation in fiber shape.

Fig. 6. A series of cylindrical map and polar projections demonstrating key differences in the fiber differentiation program after birth that results in the formation of simple star sutures throughout infancy. Short straight fibers and anterior curved straight fibers (SSFs and ACSFs) define the origin and termination of respectively, anterior secondary suture branches and pairs of posterior secondary suture branches formed sequentially in the infero-nasal (top row), infero- and supero-temporal (second row), and supero-nasal (third row) quadrants. Thus, after birth, the number of straight fibers, enantiomeric curved fiber groups, and suture branches are not identical in concentric growth shells. As a result, postnatally, there is both an intraand inter-shell variation in fiber and suture branch shape and length.
There is an intra-shell variation in fiber length that is directly related to a fiber's equatorial position.

There is an inter-shell variation in fiber length that is a radial function. There is an inter-shell variation in suture branch length that is a radial function.

\section{Suture formation after birth}

After birth there are fundamental changes in the fiber differentiation program that results in the formation of progressively more complex iterations of star sutures during infancy, adolescence and adulthood.

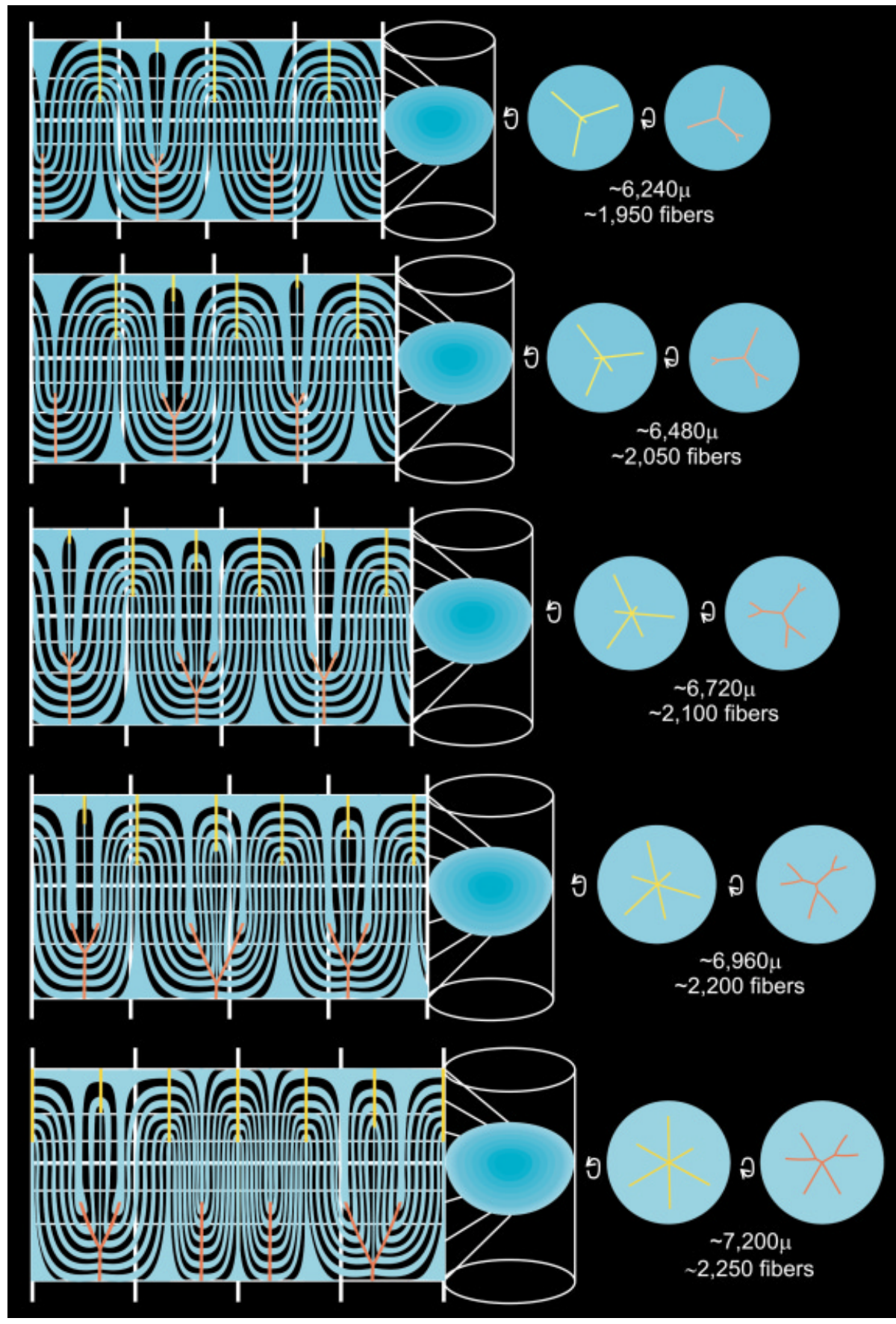



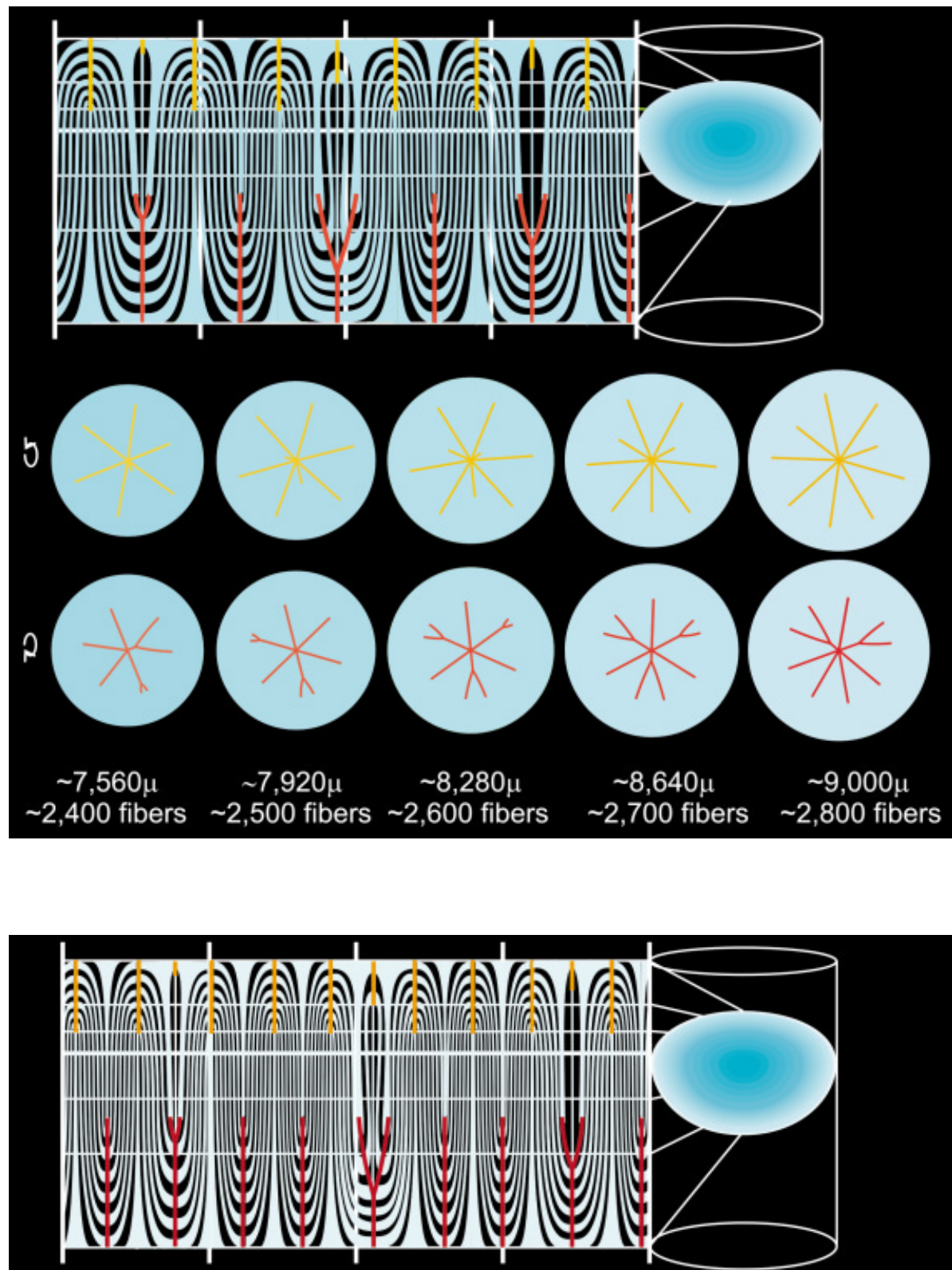

$b$

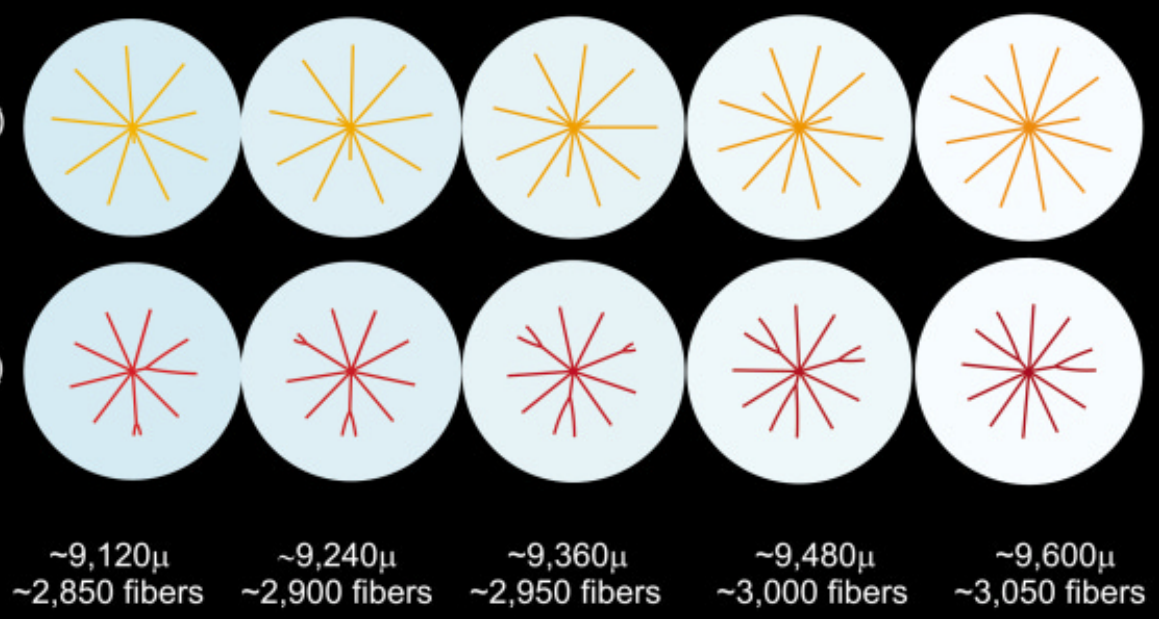

Fig. 7. Cylindrical map projections of a key stage in the development of the star (first row) suture formed throughout adolescence. These sutures are progressively more complex iterations (second and third rows, polar projections of anterior and posterior, respectively) of the simple star sutures formed through infancy. Throughout adolescence, the nine branched star sutures are formed as tertiary anterior suture branches and tertiary pairs of posterior suture branches sequentially supplement the extant primary and secondary branches.
Fig. 8. Cylindrical map projections of a key stage in the development of the complex star (first row) suture formed throughout adulthood. These sutures are progressively more complexiterations (second and third rows, polar projections, anterior and posterior, respectively) of the star sutures formed through adolescence. Similar to Fig. 7, throughout adulthood, twelve branch star sutures are formed as quaternary anterior suture and quaternary pairs of posterior suture branches sequentially supplement the extant primary, secondary and tertiary branches. 


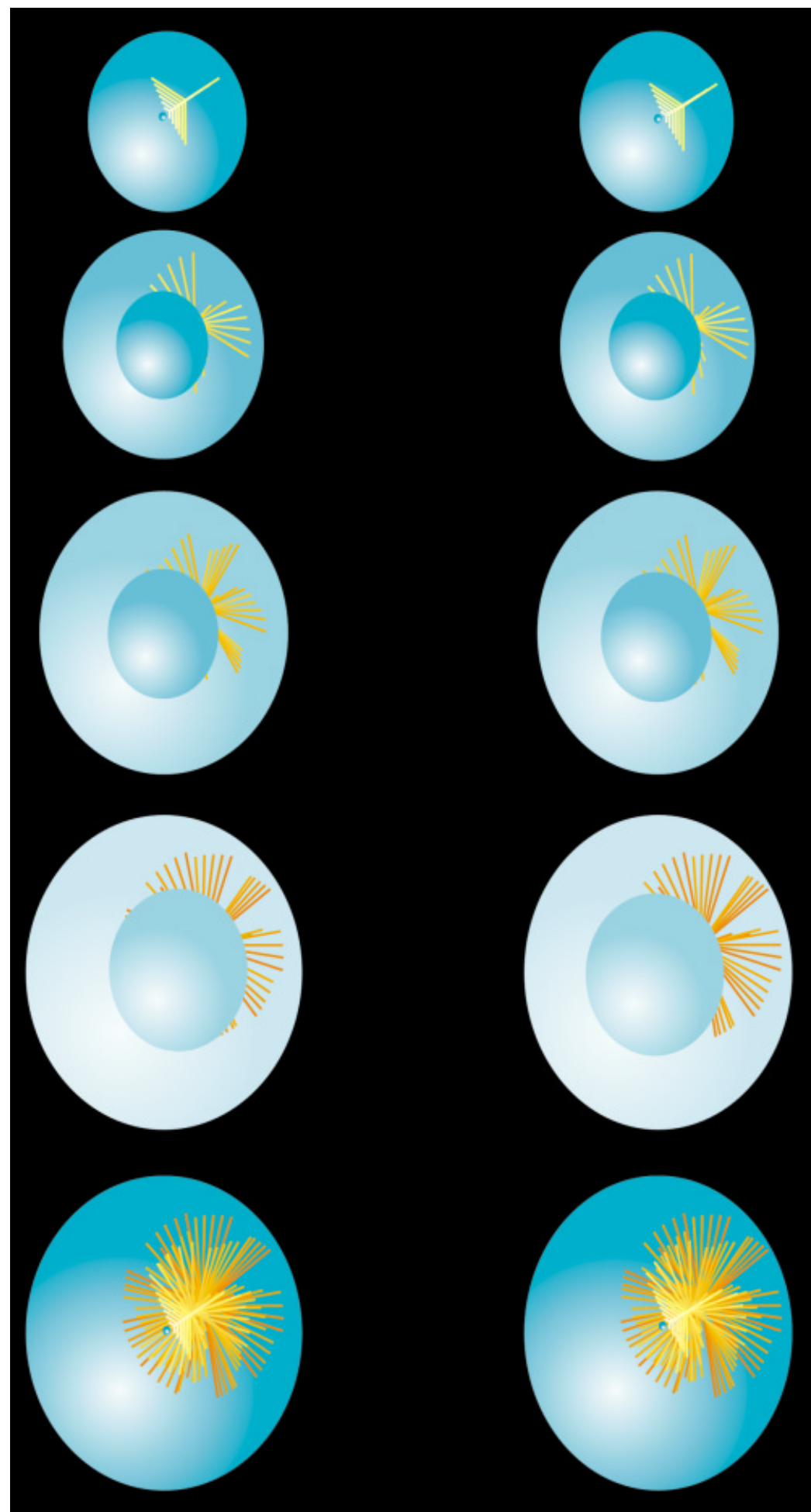

\section{Simple star suture formation}

The formation of "simple star" sutures are revealed in serial cylindrical map projections (Fig. 6). The growth shell located approximately $3,120 \mu \mathrm{m}$ from the equatorial center of a human lens consists of $\sim 1,950$ fibers. Eight of these fibers differentiated into four distinct types of straight or partially straight fibers; two ASFs, three PSFs, a short straight fiber (SSF) and two anterior curved straight fibers (ACSFs). SSFs are similar to curved fibers in that neither have ends that extend to the poles. However, like ASFs and PSFs, their entire length lies in a plane that passes through the antero-posterior axis of a lens defined by their equatorial position. ACSFs are fibers that feature a posterior end without curvature that does not extend to a pole, and an anterior end with curvature that does extend to a pole. The remaining $\sim 1900$ curved fibers in this growth shell become arranged into eight unequal groups bracketing straight fibers, unlike pre-natally, where neighboring groups of curved fibers consist of an equal number of fibers.

Thus, shortly after birth, a new, or secondary $\left(2^{\circ}\right)$ anterior suture branch, its origin defined by a SSF, and a pair of new, or $2^{\circ}$ posterior suture branches, their origins defined by ACSFs, begin to develop in relation to the extant suture branches within the infero-nasal quadrant. At the same time, the anterior ends of curved fibers that bracket PSFs are added to the extant $1^{\circ}$ anterior suture branches. Similarly, the posterior ends of the same curved fibers that bracket ASFs as a consequence of opposite end curvature, are added to the extant offset $1^{\circ}$ posterior suture branches.

As the infantile period continues, fiber differentiation produces growth shells with ten and eventually twelve straight fibers. The remaining $3,200-3,600$ curved fibers in successive growth shell become arranged into ten and eventually twelve unequal groups of curved fibers bracketing straight fibers. As a result, three $2^{\circ}$ anterior suture branch, and three pairs of $2^{\circ}$ posterior suture branches, develop sequentially in relation to the extant suture branches within the inferonasal, infero-temporal, supero-temporal and superonasal quadrants.

By the end of the infantile period the anterior suture consists of three enlarged $1^{\circ}$ branches, and three new $2^{\circ}$ suture branches one completely formed, and two partially formed. The offset simple star posterior suture consists of three pairs of new $2^{\circ}$ branches, a pair that are completely formed, two pairs that are only partially formed. All of the suture branches are arranged in a symmetrical, but non-identical, simple star suture patterns.

Fig. 9. A series of scale (4x) 3D CAD stereopairs showing the inter-shell organization of anterior suture patterns throughout gestation (top row), infancy (second row), adolescence (third row) and adulthood (fourth row). Throughout gestation, identical but larger $Y$ sutures are overlain in register to form continuous triangular suture planes extending from the embryonic nucleus to the periphery of the fetal nucleus. Throughout infancy, non-identical but larger simple star sutures are overlain in a staggered configuration extending from the fetal nucleus to the periphery of the infantile nucleus. Throughout adolescence, non-identical but larger star sutures are overlain in a staggered configuration extending from the infantile nucleus to the periphery of the juvenile nucleus. Throughout adulthood, non-identical but larger complex star sutures are overlain in a staggered configuration extending from the juvenile nucleus to the periphery of the adult nucleus. Thus, cumulatively (fifth row) from birth through adulthood, discontinuous suture planes are formed. 


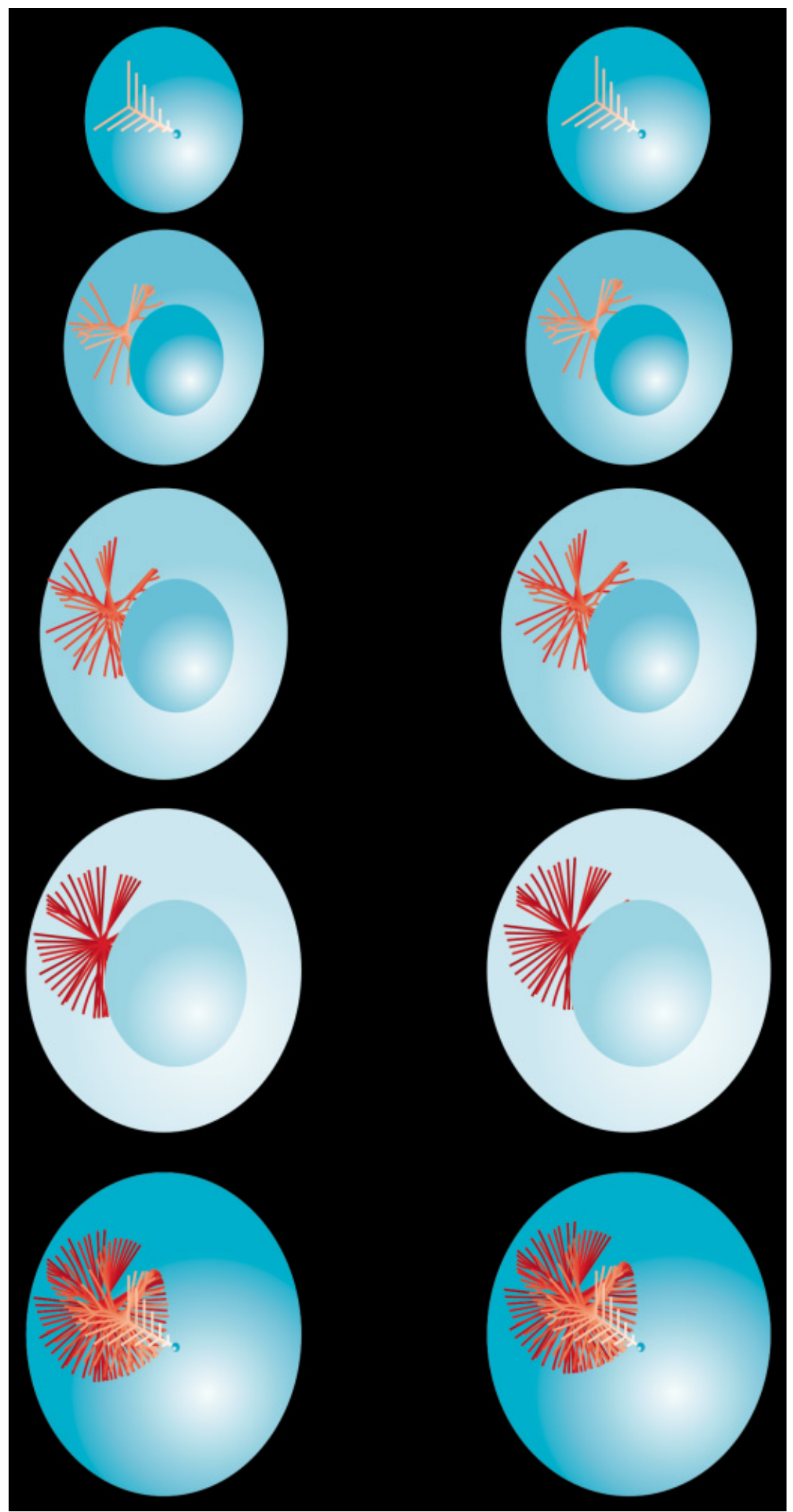

\section{Star and complex star suture formation}

The essential parameters of "star" sutures formed during adolescence, and "complex star" sutures formed throughout adulthood, are demonstrated in Figs. 7 and 8, respectively. Adolescent growth shells, located approximately $3,780-4,5000 \mu \mathrm{m}$ from the equatorial center of a human lens consists of $\sim 2,400$ $-2,800$ fibers. As the initial adolescent growth shells form, a new, tertiary $\left(3^{\circ}\right)$ anterior suture branch, and a pair of $3^{\circ}$ posterior branches begin to develop in relation to the extant $1^{\circ}$ and $2^{\circ}$ suture branches within the infero-nasal quadrant. The formation of these tertiary suture branches commences even though the formation of the second and third $2^{\circ}$ anterior branches and of the second and third pairs of $2^{\circ}$ posterior branches is still ongoing. As adolescence continues, a second and third $3^{\circ}$ anterior suture branch, and a second and third pair of $3^{\circ}$ posterior suture branches, develop sequentially in relation to the extant $1^{\circ}$ and $2^{\circ}$ suture branches within the inferotemporal, supero-temporal and supero-nasal quadrants. Thus, adolescent growth shells feature from twelve to eighteen straight fibers and comparable numbers of unequal groups of curved fibers bracketing straight fibers, with the disparity in the number of fibers/group being greater during adolescence than throughout infancy.

Adult growth shells, located approximately 4,500 $-4,8000 \mu$ from the equatorial center of a human lens consists of $\sim 2,800-3,000+$ fibers. Throughout adulthood, as growth shells form, three new, quaternary $\left(4^{\circ}\right)$ anterior suture branches, and three new pairs of $4^{\circ}$ posterior branches are formed sequentially in relation to the extant $1^{\circ}, 2^{\circ}$ and $3^{\circ}$ suture branches within the infero-nasal, infero-temporal, supero-temporal and supero-nasal quadrants. The formation of quaternary suture branches commences even though the formation of the second and third $3^{\circ}$ anterior branches and of the second and third pairs of $3^{\circ}$ posterior branches is still ongoing. Thus, adult growth shells feature from eighteen to twenty four straight fibers bracketed by comparable numbers of unequal groups of curved fibers. The disparity in the number of fibers/group is greatest during adulthood and least throughout infancy.

The different suture patterns formed during gestation, infancy, adolescence and adulthood are the anatomical basis of the zones of discontinuity revealed by slit-lamp biomicroscopy (Koretz et al., 1994; Kuszak, 1995). Throughout life anterior and posterior suture branch formation continues until their proximal ends extend to respectively 60 to 65 and -60 to $-65^{\circ}$ latitude and their distal ends extend to confluence at their respective poles. Thus, normally through middle age, the anterior sutures consist of

Fig. 10. A series of scale (4x) 3D CAD stereopairs of posterior sutures formed during the time periods as defined in Fig. 9. The net effect of life long posterior suture development also normally results in the formation of discontinuous suture planes; however, the staggering of posterior sutures is more complex than that of anterior sutures. This is the result of posterior suture development throughout life being characterized by the sequential addition of paired secondary, tertiary, and quaternary branches. 
twelve suture branches; three $1^{\circ}$ branches, three $2^{\circ}$ branches, three $3^{\circ}$ suture branches and three forming $4^{\circ}$ branches. By comparison, the offset star posterior sutures consist of three $2^{\circ}$ branches, (the $1^{\circ}$ branches are completely overlain by $2^{\circ}$ branches), six $3^{\circ}$ branches (three that are being overlain by forming pairs of $4^{\circ}$ branches), and three pairs of forming $4^{\circ}$ branches. All of the suture branches are arranged in symmetrical, but non-identical, complex star sutures. Rarely, through old age, a fifth iteration of star sutures occurs resulting in anterior and fifteen offset posterior suture branches, formed sequentially as described above. All of these suture branches are arranged in symmetrical, but non-identical, even more complex star sutures.

More often than not however, there is a compromise in the precisely defined suture formation program that results in the production of "asymmetrical" non-identical star sutures. A cylindrical map projection of a diabetic human lens (36 year old) shows that this chronic disease process has a cumulative negative effect on lens structure (Fig. 11). 3D-CAD reconstructions of early and late developing cortical cataracts suggest that their characteristic cuneiform opacities are manifestations of sutural malformation during respectively, adolescence and adulthood (Kuszak et al., 1998).

Thus, as regards star suture formation after birth, the following fiber differentiation parameters normally apply:

The anterior ends of SSFs define the proximal ends, or origins of $2^{\circ}, 3^{\circ}$ and $4^{\circ}$ anterior sutures.

The posterior ends of SSFs define the distal ends, or terminations of pairs of $2^{\circ}, 3^{\circ}$ and $4^{\circ}$ posterior sutures.

The posterior ends of ACSFs define the proximal ends, or origins of pairs of $2^{\circ}, 3^{\circ}$ and $4^{\circ}$ posterior sutures.

The anterior ends of ACSFs define the distal ends, or terminations of pairs of $2^{\circ}, 3^{\circ}$ and $4^{\circ}$ anterior sutures.

There is an intra-shell variation in curved fiber shape that is directly related to the closest straight fiber.

There is an inter-shell variation in fiber shape that is directly related to the formation of progressively more complex star sutures.

There is an intra-shell variation in fiber length that is directly related to a the formation of progressively more complex star sutures.

The inter-shell variation in fiber length is "not" a simple radial function.
The inter-shell variation in suture branch length is also "not" a simple radial function.

\section{Discontinuous suture planes and rate of anterior vs. posterior fiber elongation}

A comparison of both cylindrical and modified polar stereographic projections of gestational, infantile, adolescent and adult suture formation reveals a fundamentally important difference in the fiber differentiation program after birth. The intra and inter growth shell equatorial locations of straight fibers and/or $1^{\circ}, 2^{\circ}, 3^{\circ}$ and $4^{\circ}$ suture branches, throughout development, growth and aging are shown respectively in Figs. 5 - 8. Upon close examination it is apparent that throughout gestation, straight fibers develop in the same equatorial position. In contrast, after birth, straight fibers do not develop in the same equatorial location in concentric shells. Consequently, throughout gestation suture branches are overlain in successive growth shells thereby forming six continuous triangular suture planes extending from the embryonic nucleus to the periphery of the fetal nucleus (Figs. 9 and 10, first row). Post-natally suture branches are formed in a staggered configuration in successive growth shells thereby forming twelve, eighteen, and twenty four discontinuous suture planes extending from respectively, the periphery of the fetal nucleus to the periphery of the juvenile nucleus, from the periphery of the juvenile nucleus to the periphery of the adult nucleus, and from the periphery of the adult nucleus to the periphery of the cortex (Figs. 9 and 10, second through fourth rows). The staggering of suture branches in concentric growth shells has a positive effect on lens optical quality.

As described earlier, numerous structural studies confirm that the uniformly shaped fibers are arranged in highly ordered growth shells. However, the ends of fibers are very non-uniform in shape. Thus, their end to end arrangement to form suture branches produces naturally occurring regions of disorder aligned directly along the visual axis. In fact, by overlying suture branches in concentric growth shells, line and $Y$ suture lenses produce disordered suture planes aligned directly along the visual axis (Fig. 1). Quantitative analysis of optical quality in line and $Y$ suture lenses confirms that suture planes significantly degrade lens function

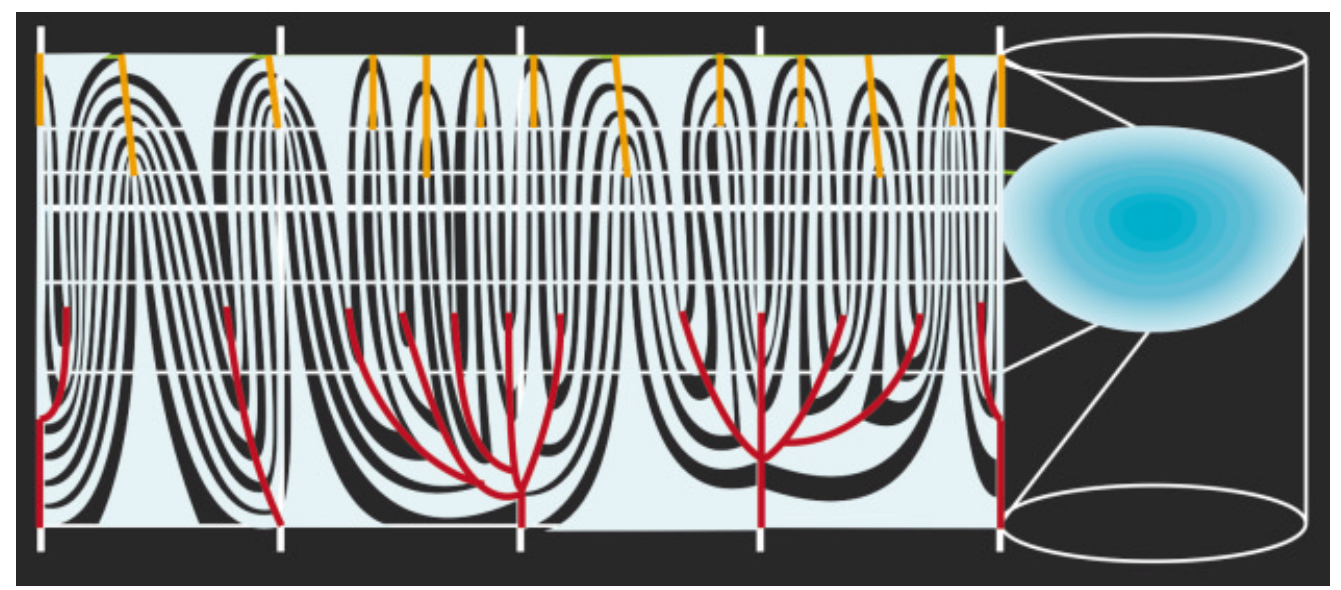

Fig. 11. Cylindrical map projection of a cortical growth shell from a $\mathbf{3 6}$ year old diabetic human lens. A comparison of this map with that of a comparably aged normal lens map (Fig. 8) suggests that the sequential addition of paired quaternary posterior sutures was compromised. It appears that two quartets of quaternary posterior suture branches had developed in this lens. 
(sharpness of focus) (Kuszak et al., 1991; Sivak et al., 1994; Kuszak etal., 1998). But. comparable studies in star suture lenses shows that the staggering of suture branches in concentric growth shells effectively minimizes the negative influence of suture planes (Kuszak et al., 1994).

It has been shown that as avian fibers elongate, they reach their posterior destination prior to their anterior destination (Bassnett and Winzenburger, 2003). Irrespective of equal or unequal rates of elongation, the cylindrical map projections suggest that the time that a fiber reaches its anterior or posterior destination is primarily a function of its equatorial location. By definition, ASFs and PSFs have one end that extends to a pole and another end which ultimately extends only to a short distance past the equator ( 2025 latitudinal degrees). Thus unless the speed of elongation anteriorly and posteriorly is widely disparate, ASFs will reach their posterior destination first, while PSFs will reach their anterior destination first. By comparison, the time that a curved fiber reaches its anterior and posterior destination, while still primarily a function of its equatorial location, would also appear to be an inverse function of its position relative to the closest straight fiber.

\section{A relationship between sutural anatomy and accommo- dation}

The direct correlation between lens sutural development throughout life and lens optical quality (sharpness of focus) strongly suggests that this structural parameter may also have a key role in accommodation. Birds and reptiles have powerful mechanisms that compress the lens to accommodate for near vision. These lenses lack suture branches. In contrast, most vertebrate lenses have suture branches overlain in concentric growth shells to form continuous suture planes. These lenses have minimal accommodative range. The mammalian mode of accommodation is more advanced in primates. These lenses have suture branches offset in successive growth shells to thereby form discontinuous suture planes (Koretz et al., 1994; Kuszak, 1995). Preliminary results of ongoing studies reveal that the end segments of fibers from lenses with a broad range of accommodation are flat and smooth. With the use of an ex-vivo mechanical stretching apparatus that simulates changes in lens shape during accommodation and un-accommodation (Glasser and Campbell, 1998), it may be possible to show that these end segments interlace with each other in a un-accommodated state. By comparison, the end segment of fibers from lenses with a small range of accommodation are large and irregular, suggesting that these end segments are not conducive to coordinated changes in lens shape necessary for accommodation.

\section{The contribution of major fiber proteins to sutural de- velopment and growth}

In vertebrate lenses MP26, gap junction (GJ) connexins, and MP 19, are the major fiber intrinsic membrane proteins. The major intrinsic protein (MIP), or aquaporino (AQP0), is generally described as constituting more than $60 \%$ of the total fiber membrane protein (Steele, Jr. et al., 2000; Zhou et al., 2002). MIP forms either thin symmetric or asymmetric junctions (TSJs and TAJs) also referred to as square array membrane. Square array membrane is likely to serve one of two functions as a consequence of their arrangement between fibers. The AQP0s can serve as single membrane water transport channels that remove water from the extracellular space (Broekhuyse et al., 1976; Ehring et al., 1992; King and Agre, 1996; Fotiadis et al., 2000), or the close association of apposed undulating membrane in TAJs can subserve an adhesive function (Fotiadis et al., 2000). In the lens, cell-to-cell communication is mediated by pleomorphic gap junctional domains and membrane gateways permeable to ions, water and small metabolites (Kumar and Gilula, 1996; Dunia et al., 1998). The connexins (Cxs; (Bruzzone etal., 1996; Kumar and Gilula, 1996)) that form the lens GJ communicating channels, display a variety of expression patterns, channel regulation and post-translational modifications during differentiation and aging of the lens cells (Goodenough, 1992; Benedetti et al., 1996). Lens epithelial GJs consist mainly of .1 connexin (Cx.1 or Cx43; (Musil and Goodenough, 1991)). Cortical fiber GJs consist of Cx.3 (or Cx46) and Cx.8 (or Cx50) often coexisting in the same junctional plaques (Paul et al., 1991; Evans et al., 1992; Lin et al., 1997). MP 19 (also referred to as MP 17, MP 18 and MP20 in the literature) has also been described as the most abundant intrinsic membrane proteins of lens fiber cells (Steele, Jr. et al., 2000). However, unlike MIP or the connexins, MP 19 bears no striking resemblance to any other reported protein family and to date has no defined structural or functional role. Both MIP and MP 19 co-localize with GJs in distinct regions of the lens (TenBroek et al., 1992; TenBroek et al., 1994). Thus, it has been proposed that both MIP and MP 19 play some role in GJ formation, maintenance or organization.

While it is well documented and irrefutable that all vertebrate lenses contain the above described major fiber proteins, a review of the literature suggests that their density and distribution varies is species specific varying along fiber length, and as a function of fiber depth (age). GJs primarily conjoin the mid-segment of fibers, or those segments of fibers not involved in sutures (Fitzgerald, 1986; Kuszak and Costello, 2002). Chick, rat, mouse, frog and human fibers have respectively $65,33,15,12$ and $5 \%$ of the midsegment of cortical fibers are specialized as GJs. In a study characterizing the structure of lenses from MIP26/Aqp0 deficient mice (Al-Ghoul et al., 2003), it has been shown that wild type mouse cortical fibers had square array membrane randomly distributed along anterior and posterior end segments whereas 15\% of the fiber mid-segments were specialized as GJs. In contrast, square array membrane was essentially absent In +/- mice cortical fibers. However, while these fibers had a similar density of GJs between the mid-segment of fibers to that in $+/+$ mice, these junctions had a significantly different size range. Cortical fibers in -/- mice, had a complete lack of square array membrane, featured only $7 \%$ of the mid-segment of fibers specialized as GJs, and these GJs again had a size distribution that was significantly different than in heterozygotes. These findings are consistent with reports that both MIP and MP 19 have been shown to co-localize with GJs in distinct regions of lenses (TenBroek etal., 1992; TenBroek etal., 1994; Dunia et al., 2001). Thus, the function of these proteins is probably coordinated during fiber development.

Further review of the literature reveals the following; primate lenses, have a broad accommodative range, complex suture patterns, a low density of fiber GJs, and a high density of TAJs. Avian lenses, also have a broad accommodative range, but have the simplest of suture patterns, the highest density of fiber GJs, and the lowest density of TAJs of all vertebrate lenses. Non-primate mammalian lenses, have a very limited accommodative range, 
suture patterns of intermediate complexity, and intermediate density of both fiber GJs and TAJs. Thus, additional studies to confirm if a relationship exists between accommodative ability and the density and distribution of the major fiber membrane proteins along fiber length and as a function of development, growth and age are warranted.

\section{Summary}

It is clear from all of the above that the most apparent structural consequence of lens differentiation is not merely the transformation of a cuboidal epithelial cell into an exceedingly long crescent shaped fiber. Instead, fiber differentiation results in the formation of fibers with variable shapes and length. The precise end to end arrangement of fibers within and between concentric growth shells form sutures that are not identical in all lenses. There are four distinct types of lens sutures with increasing complexity that exert a quantifiable negative influence on lens optical quality. These different suture types might also be a factor in establishing and maintaining the range of accommodation. In summary, lenses are a prime example of form following function and malformation leading to malfunction.

\section{Acknowledgements}

The authors wish to acknowledge the expert technical assistance of Kurt L. Peterson, Layne A. Novak, and Mike Mazurkiewicz. This work supported by NIH grant \#EY06642 to JRK.

\section{References}

AL-GHOUL K.J. and COSTELLO M.J. (1997). Light microscopic variation of fiber cell size, shape and ordering in the equatorial plane of bovine and human lenses. Mol. Vision 3: 2.

AL-GHOUL K.J., KIRK T., KUSZAK A.J., ZOLTOSKI R.K., SHIELS A. and KUSZAK JR. (2003). Lens structure in MIP-deficient mice. Anat. Rec. 273A: 714-730.

AL-GHOUL K.J., NOVAK L.A., PETERSON K.L. and KUSZAK J.R. (1999). Internalization of posterior subcapsular cataracts (PSCs) in Royal College of Surgeons (RCS) rats. I. Morphological characterization. Mol. Vision 5: 6.

BASSNETT S. and WINZENBURGER P.A. (2003). Morphometric analysis of fibre cell growth in the developing chicken lens. Exper. Eye Res. 76: 291-302.

BENEDETTI E.L., DUNIA I., DUFIER J.L., YIT KIM SENG and BLOEMENDAL H. (1996). Plasma membrane-cytoskeleton complex in the normal and cataractous lens. In: The Cytoskeleton (Hesketh JE, Pryme IF, eds), pp 451-518. London: JAI Press Inc.

BROEKHUYSE R.M., KUHLMAN E.D. and STOLS A.L. (1976). Lens membranes II. Isolation and characterization of the main intrinsic polypepetide (MIP) of bovine lens fiber membranes. Exper. Eye Res. 23: 365-371.

BRUZZONE R., WHITE T.W., GOODENOUGH D.A. (1996). The cellular internet: online with connexins. BioEssays 18: 709-718.

COHEN A.I. (1965). The electron microscopy of the normal human lens. Invest. Ophthalmol. 4: 433-446.

DUKE-ELDER S. (1970). The refractive media: The lens. In: Systems of Ophthalmology (Duke-Elder S, ed), pp 309-324. St. Louis, Mo.: The C.V. Mosby Co.

DUNIA I., RECOUVREUR M., NICOLAS P., KUMAR N., BLOEMENDAL H. and BENEDETTI E.L. (1998). Assembly of connexins and MP26 in lens fiber plasma membranes studied by SDS-fracture immunolabeling. J. Cell. Sci. 111:2109-2120.

DUNIA I., RECOUVREUR M., NICOLAS P., KUMAR N.M., BLOEMENDAL H. and BENEDETTIE.L. (2001). Sodium dodecyl sulfate-freeze-fracture immunolabeling of gap junctions. In: Methods in Molecular Biology (Bruzzone R, Giaume C, eds), pp 33-55. Totowa, N.J.: Humana Press, Inc.

EHRING G.R., LAGOS N., ZAMPIGHI G.A. and HALL J.E. (1992). Phosphorylation modulates the voltage dependence of channels reconstituted from the major intrinsic protein of the lens fiber membranes. J. Membr. Biol. 126: 75-88.
EVANS C.W., EASTWOOD S., RAINS J.W., GRUIJTERS T.M., BULLIVANT S. and KISTLER J. (1992). Gap junction formation during development of the mouse. Eur. J. Cell. Biol. 60: 243-249.

FITZGERALD P.G. (1986). The main intrinsic polypeptide and intercellular communication in the ocular lens. In Development of order in the visual system (Hilfer RS, Sheffield JB, eds), pp 61-96. New York: Springer-Verlag.

FOTIADIS D., HASLER L., MULLER D.J., STAHLBERG H., KISTLER J. and ENGEL A. (2000). Surface tongue-and-groove contours on lens MIP faciitate cell-to-cell adherence. J. Mol. Biol. 300: 779-789.

GLASSER A. and CAMPBELL M.C. (1998). Presbyopia and the optical changes in the human crystalline lens with age. Vision Res. 38: 209-229.

GOODENOUGH D.A. (1992). The crystalline lens. A system networked by gap junctional intercellular communication. Sem. Cell Biol. 3: 49-58.

HARDING C.V., REDDAN J.R., UNAKAR N.J. and BAGCHI M. (1971). The control of cell division in the ocular lens. Int. Rev. Cytol. 31: 215-300.

KELZ M.B., KUSZAK J.R., YANG Y., MA W., STEFFEN C., AL-GHOUL K.J., ZHANG Y., CHEN J., NESTLER E.J. and SPECTOR A. (2000). Delta FosB induced cataract. Invest. Ophthal. Vis. Sci. 41: 3523-3538.

KING L.S. and AGRE P. (1996). Pathophysiology of the aquaporin water channels. Annu. Rev. Physiol. 58: 619-648.

KORETZ J.F., COOK C.A. and KUSZAK J.R. (1994). The zones of discontinuity in the human lens: Development and distribution with age. Vision Res. 34: 29552962.

KUMAR N.M. and GILULA N.B. (1996). The gap junction communication channel. Cel/84: 381-388.

KUSZAK J.R. (1995). Development of sutures in the lens. Prog. Retina/ \& Eye Res. 14: $567-591$.

KUSZAK J.R., AL-GHOUL K.J. and COSTELLO M.J. (1998). Pathology of AgeRelated Human Catarcts. In Duane's Clinical Ophthalmology (Tasman W, Jaeger EA, eds), pp 1-14. Philadelphia: Lippincott, Williams and Wilkins.

KUSZAK J.R., AL-GHOUL K.J., NOVAK L.A., PETERSON.K.L., HERBERT K.L., and SIVAK J.G. (1999). The internalization of posterior subcapsular cataracts (PSCs) in Royal College of Surgeons (RCS) rats. II. The inter-relationship of optical quality and structure as a function of age. Mol. Vision 5: 7.

KUSZAK J.R., ALCALA J. and MAISEL H. (1980). The surface morphology of embryonic and adult chick lens-fiber cell. Am. J. Anat. 159: 395-410.

KUSZAK J.R. and COSTELLO M.J. (2002). Embryology and anatomy of the lens. In Duane's Clinical Ophthalmology (Tasman W, Jaeger E, eds), pp 1-19. Philadelphia, PA: J.B. Lippincott Co.

KUSZAKJ.R., ENNESSER C.A., BERTRAM B.A., IMHERR-MCMANNISS., JONESRUFER L.S. and WEINSTEIN R.S. (1989). The contribution of cell-to-cell fusion to the ordered structure of the crystalline lens. Lens Eye Toxic. Res. 6: 639-673.

KUSZAK J.R., MACSAI M.S., BLOOM K.J., RAE J.L. and WEINSTEIN R.S. (1985). Cell-to-cell fusion of lens fiber cells in situ: correlative light,scanning electron microscopic, and freeze-fracture studies. J. Ultrastruct. Res. 93: 144-160.

KUSZAK J.R., PETERSON K.L. and BROWN H.G. (1996). Electron microscopic observations of the crystalline lens. Microsc. Res. Tech. 33: 441-479.

KUSZAK J.R., PETERSON K.L., HERBERT K.L. and SIVAK J.G. (1994). The interrelationship of lens anatomy and optical quality. II. Primate lenses. Exper. Eye Res. 59: 521-535.

KUSZAK J.R. and RAE J.L. (1982). Scanning electron microscopy of the frog lens. Exper. Eye Res. 35: 499-519.

KUSZAK J.R., SIVAK J.G., HERBERT K.L., SCHEIB S., GARNER W. and GRAFF G. (2000). The relationship between rabbit lens optical quality and sutural anatomy after vitrectomy. Exper. Eye Res. 71: 267-282.

KUSZAK J.R., SIVAK J.G., MORAN K.L., SCHEIB S.A., GARNER W.H., KE T.L., HELLBERG M.R. and GRAF G. (2002). Suppression of post-vitrectomy lens changes in the rabbit by novel benzopyranyl esters and amides. Exper. Eye Res. 75: 459-473.

KUSZAK J.R., SIVAK J.G. and WEERHEIM J.A. (1991). Lens optical quality is a direct function of lens sutural architecture. Invest. Ophthal. Vis. Sci. 32, No. 7: 2119-2129.

LIN J.S., FITZGERALD S., DONG Y., KNIGHT C., DONALDSON P. and KISTLER J. (1997). Processing of gap junction protein connexin 50 in the ocular lens is accomplished by calpain. Eur. J. Cell. Biol. 73: 141-149. 
MUSIL L.S. and GOODENOUGH D.A. (1991). Biochemical analysis of Connexin 43 intracellular transport, phosphorylation, and assembly into gap junctional plaques. J. Cell. Biol. 115: 1357-1374.

O'RAHILLY R. and MEYER D.B. (1959). The early development of the eye in the chick Gallus Domesticus (stages 8 to 25). Acta Anatomica 36: 20-58.

PAUL D.L., EBIHARA L., TAKEMOTO L.J., SWENSON K.I. and GOODENOUGH D.A. (1991). Cx46, a novel lens gap junction protein, induces voltage-gated currents in nonjunctional plasma membranes of Xenopus oocytes. J. Cell. Biol. 115: 1077-1089.

PRIOLO S., SIVAK J.G. and KUSZAK J.R. (2000). Effect of experimentally induced ametropia on the morphology and optical quality of the avian crystalline lens. Invest. Ophthal. Vis. Sci. 41: 3516-3522.

RAE J.L., TRUITT K.D. and KUSZAK J.R. (1982). A simple fluorescence technique for light microscopy of the crystalline lens. Curr. Eye Res. 2: 1-5.

RAE J.L., TRUITT K.D. and KUSZAK J.R. (1983). The use of procion dyes for light microscopy of the frog lens. Invest. Ophthal. Vis. Sci. 24: 1167-1171.

RAFFERTY N.S. (1985). Lens Morphology. In The Ocular Lens: Structure, Function and Pathology. (Maisel H, ed), pp 1-60. New York: Marcel Dekker, Inc.

RAFFERTY N.S. and ESSON E.A. (1974). An electron microscope study of adult mouse lens: some ultrastructural specializations. J. Ultrastruct. Res. 46: 239253.
SIVAK J.G., HERBERT K.L., PETERSON K.L. and KUSZAK J.R. (1994). The InterRelationship of Lens Anatomy and Optical Quality. I. Non-Primate Lenses. Exper. Eye Res. 59: 505-520.

SMELSER G.K. 1965. Embryology and morphology of the lens. In Symposium on the lens (Harris JE, ed), pp 22-34. Saint Louis: C.V. Mosby Co.

STEELEE.C.,JR.,WANG J.-H.,LOW.-K. SAPERSTEIND.A.,LIX.L., and CHURCH R.L. (2000). Lim2To3 transgenic mice establish a causative relationship between the mutation identified in the Lim2gene and cataractogenesis in the To3mouse mutant. Mol. Vis. 6: 85-94.

TENBROEK E.M., ARNESON M., JARVIS L. and LOUIS C. (1992). The distribution of the fiber cell intrinsic membrane proteins MP20 and connexin46 in the bovine lens. J. Cell Sci. 103: 245-257.

TENBROEK E.M., JOHNSON R., and LOUIS C.F. (1994). Cell-to-cell communication in a differentiating ovine lens culture system. Invest. Ophthal. Vis. Sci. 35:215-228.

TROKEL S. (1962). The physical basis for transparency of the crystalline lens. Invest Ophthalmol. 1: 493-501.

WILLEKENS B. and VRENSEN G. (1985). Lens fiber organization in four avian species: A scanning electron microscopy. Tissue Cel/17 (3): 359-377.

ZHOU L., CHEN T. and CHURCH R.L. (2002). Temporal expression of three mouse lens fiber cell membrane protein genes during early development. Mol. Vis. 8: 143-148. 